\title{
WORLD FEDERATION OF OCCUPATIONAL THERAPISTS' POSITION STATEMENT ON TELEHEALTH
}

\author{
WORLD FEDERATION OF OCCUPATIONAL THERAPISTS \\ ForrestField Western Australia, Australia
}

\section{ABSTRACT}

The purpose of this document is to state the World Federation of Occupational Therapists' (WFOT) position on the use of telehealth for the delivery of occupational therapy services. Telehealth is the use of information and communication technologies (ICT) to deliver health-related services when the provider and client are in different physical locations. Additional terms used to describe this service delivery model include: tele-occupational therapy, telerehabilitation, teletherapy, telecare, telemedicine, and telepractice, among other terms. Telehealth may be used by occupational therapy practitioners for evaluation, intervention, monitoring, supervision, and consultation (between remote therapist, client, and/or local health-care provider) as permitted by jurisdictional, institutional, and professional regulations and policies governing the practice of occupational therapy. Occupational therapy services via telehealth should be appropriate to the individuals, groups and cultures served, and contextualized to the occupations and interests of clients. Important considerations related to licensure/registration, collaboration with local occupational therapists, client selection, consent to treat, professional liability insurance, confidentiality, personal and cultural attributes, provider competence/standards of care, reimbursement/payer guidelines, and authentic occupational therapy practice are discussed.

Key words: Occupational therapy, telecare, telehealth, tele-occupational therapy, telerehabilitation, World Federation of Occupational Therapists

\section{INTRODUCTORY STATEMENT OF PURPOSE AND DEFINITIONS}

The purpose of this paper is to state the World Federation of Occupational Therapists' (WFOT) position on the use of telehealth for the delivery of occupational therapy services.

Telehealth is the use of information and communication technologies (ICT) to deliver health-related services when the provider and client are in different physical locations. While various terms are used to describe this remote service delivery model, the term telehealth best aligns with the overarching nomenclature used by health officials and policy makers and aptly describes the full scope of occupational therapy practice (e.g., health promotion, habilitation, rehabilitation, etc.). Additional terms used to describe this service delivery model include: teleoccupational therapy, telerehabilitation, teletherapy, telecare, telemedicine, and telepractice, among other terms. WFOT defines occupational therapy as a "clientcentred health profession concerned with promoting health and well-being through occupation" (p. 3). By virtue of this definition, occupational therapists promote health regardless of the context in which they practice.

The term 'telehealth' can refer to synchronous (realtime) interactions between the therapist and client (e.g., videoconference, remote monitoring, virtual interactions using applications ('apps') and gaming technologies); and/ or asynchronous (i.e., "store-and-forward") transmission of data (e.g., video, photos, electronic mail) by the provider and/or the client. In some circumstances, telehealth may be selectively employed as part of a hybrid service delivery model that incorporates both in-person interaction and telehealth. Telehealth may be used for evaluation, intervention, monitoring, supervision, and consultation (between remote therapist, client, and/or local health-care provider) as permitted by jurisdictional, institutional, and professional regulations and policies governing the practice of occupational therapy 


\section{STATEMENT OF POSITION}

The WFOT's mission to develop occupational therapy worldwide presupposes access to services that are contextualized to local culture, resources, and occupations. The WFOT affirms:

Occupational therapy services provided via telehealth should meet the same standards of care as services delivered inperson and comply with all jurisdictional, institutional, and professional regulations and policies governing the practice of occupational therapy.

Occupational therapy services are ideally delivered by locally trained and culturally competent occupational therapists. The United Nation's Convention on the rights of persons with disabilities ${ }^{2}$ asserts that State Parties shall "organize, strengthen and extend comprehensive habilitation and rehabilitation services and programmes" and that these services and programmes be "available to persons with disabilities as close as possible to their own communities, including in rural areas" (Article 26 - Habilitation and rehabilitation). Telehealth may improve access to services within clients' communities and 'strengthen and extend comprehensive habilitation and rehabilitation services' via the transfer of knowledge and skills from remote specialists to local health-care providers through consultation and mentoring relationships.

Telehealth is an appropriate delivery model for occupational therapy services when in-person services are not possible, practical, or optimal for delivering care and/or when service delivery via telehealth is mutually acceptable to the client and provider. Telehealth can also be part of a hybrid model wherein some occupational therapy services are delivered to a client in-person, and some occupational therapy services are delivered at a distance.

Occupational therapy services via telehealth should be appropriate to the individuals, groups and cultures served, and contextualized to the occupations and interests of clients.

Telehealth competencies and guidelines should be defined via collaborations between occupational therapists, educators, member associations, and other societal stakeholders.

\section{STATEMENT OF SIGNIFICANCE OF THE POSITION TO SOCIETY}

Telehealth connects clients with occupational therapists through information and communication technologies, thereby improving access to services for underserved populations when in-person encounters are not possible or practical. Telehealth can address education gaps for local providers, and contribute to initiatives to address shortages of occupational therapy personnel.

The World Report on Disabilities ${ }^{3}$ co-produced by the World Health Organization and World Bank affirmed the efficacy of telehealth for the delivery of rehabilitation services (i.e., telerehabilitation) stating its use "leads to similar or better clinical outcomes when compared to conventional interventions" (p. 119). Studies cited in the report demonstrate that telehealth yields comparable outcomes to rehabilitation services delivered in-person. Telehealth technologies also facilitate remote training and support of health-care providers and "sharing professional expertise between countries, as well as at critical times such as in the aftermath of a disaster" ( $p$. 119).

\section{Challenges And STRATEGies}

The WFOT asserts that telehealth use by occupational therapists should adhere to the WFOT Code of Ethics ${ }^{4}$ and comply with jurisdictional, institutional, and professional regulations and policies governing occupational therapy practice. Important considerations include:

- Licensure/Registration - Therapists shall comply with professional licensure/registration requirements. The WFOT's document, Working as an OT in Another Country ${ }^{5}$ provides an overview of registration, practice, and membership requirements in the majority of WFOT Member Organisations.

- Collaboration with Local Occupational Therapists - Telehealth providers are encouraged to seek opportunities to collaborate with and promote local occupational therapy providers, organizations, educational institutions, and/or associations in the interest of cohesive, relevant, and sustainable services.

- Client Selection - Therapists should use clinical reasoning to determine the appropriateness of telehealth use based on individual client situations (e.g., client's diagnosis and impairments, nature of the occupational therapy interventions to be provided, client's ability to access technologies, etc.). Telehealth should therefore not be used to avoid in-person services when indicated by client-specific needs, nor be used by therapists to avoid contact with clients on the basis of discrimination. (See also WFOT's document, Clientcentredness in Occupational Therapy ${ }^{6}$ )

- Consent to Treat - Therapists shall inform clients about the nature of the occupational therapy services 
to be provided, risks, benefits, alternate treatment options, and any limits to protection of privacy, security, and confidentiality of personal health information associated with the technology.

- Professional Liability Insurance - Occupational therapists should comply with jurisdictional, institutional, and professional requirements for maintaining professional liability insurance. Occupational therapists should confirm coverage of professional liability insurance for the geographic areas served.

- Confidentiality - Users of telehealth are obligated to employ mechanisms to ensure confidentiality for synchronous and stored client data in compliance with jurisdictional, institutional, and professional regulations and policies governing occupational therapy practice.

- Personal and Cultural Attributes - Therapists should follow principles outlined in the WFOT's document, Guiding Principles on Diversity and Culture $^{7}$ and the Diversity and Culture ${ }^{8}$ position statement.

- Provider Competence/Standard of Care Therapists must maintain professional competency, acquire competency using telehealth technologies, ensure client safety, and adhere to ethical principles of practice.

- Reimbursement/Payer Guidelines - Therapists must adhere to reimbursement requirements and accurately represent the services delivered through telehealth.

- Authentic Occupational Therapy Practice - The WFOT endorses practice that is client-centred and occupation-centred, and which portrays the breadth of the profession.

\section{CONCLUSION}

Telehealth can be an appropriate service delivery model for occupational therapy, and may improve access to occupational therapy services.

Approved by: The WFOT Council Meeting, Japan, June 2014; available in the Resource Centre on the WFOT website at http://www.wfot.org/ResourceCentre.aspx

\section{REFERENCES}

1. World Federation of Occupational Therapists [WFOT]. (2013). Definitions of occupational therapy from member organisations (revised 2013 October). Retrieved from http://www.wfot.org/ResourceCentre.aspx

2. United Nations. (2006). Convention on the rights of persons with disabilities. Retrieved from http://www. un.org/disabilities/convention/conventionfull.shtml

3. World Health Organization \& World Bank. (2011). World report on disability. Retrieved from http://whqlibdoc.who. int/publications/2011/9789240685215_eng.pdf

4. World Federation of Occupational Therapists [WFOT]. (2005). Code of Ethics (revised 2005). Retrieved from http://www.wfot.org/ResourceCentre.aspx

5. World Federation of Occupational Therapists [WFOT]. (2013). Working as an occupational therapist in another country. Retrieved from http://www.wfot.org/ ResourceCentre.aspx

6. World Federation of Occupational Therapists [WFOT]. (2010). Client-centredness in occupational therapy. Retrieved from http://www.wfot.org/ResourceCentre. aspx

7. World Federation of Occupational Therapists [WFOT]. (2009). Guiding principles on diversity and culture. Retrieved from http://www.wfot.org/ResourceCentre/ tabid/132/did/306/Default.aspx

8. World Federation of Occupational Therapists [WFOT]. (2010). Diversity and Culture. Retrieved from http://www. wfot.org/ResourceCentre.aspx 
